\title{
Nonlinear Neural System for Active Noise Controller to Reduce Narrowband Noise
}

\author{
Minh-Canh Huynh $\mathbb{I D}^{1,2}$ and Cheng-Yuan Chang $\mathbb{D}^{2}$ \\ ${ }^{1}$ Department of Electrical Engineering, Eastern International University, Binhduong 590000, Vietnam \\ ${ }^{2}$ Department of Electrical Engineering, Chung Yuan Christian University, Taoyuan 320, Taiwan \\ Correspondence should be addressed to Cheng-Yuan Chang; ccy@cycu.edu.tw
}

Received 30 January 2021; Revised 30 March 2021; Accepted 18 May 2021; Published 30 May 2021

Academic Editor: Zhao Kang

Copyright $\odot 2021$ Minh-Canh Huynh and Cheng-Yuan Chang. This is an open access article distributed under the Creative Commons Attribution License, which permits unrestricted use, distribution, and reproduction in any medium, provided the original work is properly cited.

\begin{abstract}
Noise in a dynamic system is practically unavoidable. Today, such noise is commonly reduced using an active noise control (ANC) system with the filtered-x least mean square (FXLMS) algorithm. However, the performance of the ANC system with FXLMS algorithm is significantly impaired in nonlinear systems. Therefore, this paper develops an efficient nonlinear adaptive feedback neural controller (NAFNC) to eliminate narrowband noise for both linear and nonlinear ANC systems. The proposed controller is implemented to update its coefficients without prior offline training by neural network. Hence, the proposed method has rapid convergence rate as confirmed by simulation results. The proposed work also analyzes the stability and convergence of the proposed algorithm. Simulation results verify the effectiveness of the proposed method.
\end{abstract}

\section{Introduction}

Noise is everywhere and affects us constantly. The usual approach to reduce noise uses sound-proof or sound-absorbing materials, called passive noise reduction, having the disadvantage of requiring bulky materials and being ineffective in cancelling low-frequency noise. The active noise control (ANC) method, introduced by Lueg in 1936 [1], is built by adding a secondary source to eliminate the undesired noise by the superposition principle. An ANC system emits antinoise that has an equal amplitude but with opposite phase to the primary noise source. Two sound waves from both primary and secondary sources interfere with each other, resulting in both noise cancellation and very efficient low-frequency operation [2].

In recent years, several ANC approaches have been applied to vibration and noise reduction for vehicles. Gäbel et al. proposed a multichannel active control system to reduce vehicle interior noise on the road. The proposed algorithm reduced the level of structural vibration and vehicle interior noise under the operating conditions [3]. Wang et al. proposed active control using the discrete wavelet transform (DWT) based FXLMS algorithm with a piezoelectric feedback system to cope with the stationary and nonstationary noises in a simplified vehicle cavity model [4]. Guo et al. improved fuzzy control algorithm based on piezoelectric ceramic materials and proposed the variable stepsize median-LMS algorithm for the vehicle interior noise $[5,6]$. Thereby, it can be seen that the ANC system has made positive contributions to life.

Usually, the ANC systems can be divided into two categories, the broadband and the narrowband ANC systems. The narrowband ANC (NANC) system includes two methods: feedforward and feedback ways $[7,8]$. The feedback ANC method applies error signal and then synthesizes the reference signal, which reduces estimated noise $[9,10]$. On the other hand, a feedforward system measures the noise using a reference sensor and thus is capable of cancelling broadband noise [11]. The FXLMS algorithm is widely used in the ANC systems to tune the coefficients to achieve the best noise reduction. The variable step-size method for FXLMS algorithm was proposed in [12] for a typical NANC system which supports rapid convergence. Chang et al. presented a filter-bank in a parallel-structure NANC for 
secondary path modelling to enhance ANC performance [13-15]. Shin et al. proposed a sequential selection-normalized subband adaptive filter with variable step size, which involved low computational complexity and had an efficient convergence speed [16]. However, the ANC system is usually influenced by acoustic environment such as the varying time, temperature, and other factors. In addition, the ANC system is also affected by the stability of sensors, actuators, and amplifiers, which may degrade the performance of the linear ANC with the FXLMS algorithm [17].

Consequently, many studies proposed nonlinear ANC designs. The adaptive recursive Volterra controller and adaptive bilinear controller have been used with nonlinear systems [18-20]. With respect to fuzzy controllers, the fuzzy ANC system was proposed in [21]. Aluclu et al. also built a fuzzy logic model to restrain noise in workplace [22]. Chang et al. developed the fuzzy filtered-U algorithm to avoid unstable poles, which may occur in the conventional filteredU based ANC system [23]. Besides, combining fuzzy and neural network schemes, several nonlinear ANC systems have also been presented [24-28]. The multilayer neural networks have been proposed for nonlinear ANC systems [29-32]. Halimeh et al. also introduced a neural networkbased approach to the problem of nonlinear acoustic echo cancellation (NLAEC) [33]. Zhang mentioned a nonlinear neural adaptive controller integrated with evaluation algorithm to attenuate noises in nonlinear systems [34]. Thai et al. used an adaptive variable step-size neural controller in nonlinear feedback ANC systems, resulting in the reduction of noise that was transmitted over nonlinear paths and thereby improving the performance [35]. Notably, Thai et al. proposed a fast learning algorithm for neural networks that was based on the FXLMS algorithm without offline training.

Based on the above analysis, the FXLMS algorithm-based adaptive neural controller can update parameters online without offline training in advance. However, the feedforward neural controller approach is less effective than the feedback system $[36,37]$ because the feedback path allows the neural network to learn, recognize, and generate the time-varying patterns. The delay in the feedback connection stores values from the previous time step. These values can be used in the current time step [37, 38]. This research develops a nonlinear adaptive feedback neural controller (NAFNC) that can be adapted in nonlinear conditions to enhance the performance of the NANC system. The advantages of this paper can be recapitulated as follows:

(1) The parameters of the NAFNC are updated online using the FXLMS algorithm

(2) The convergence and stability of the proposed method are ensured by analyzing the Lyapunov function

(3) The obtained results of the simulations were compared with the works in [35], revealing improved performance of the proposed method

(4) Simulation results verify the effectiveness of the proposed method in both linear and nonlinear ANC systems
This paper is organized as follows. Section 2 analyzes conventional ANC methods. Section 3 provides the proposed method. Section 4 presents simulation results. Section 5 draws conclusions.

\section{Conventional Methods Analysis}

This section analyzes the algorithm of the conventional methods in ANC systems.

2.1. Offline Modelling to the Secondary Path. The ANC system with the FXLMS algorithm must estimate the secondary path to update the coefficients of the adaptive filter. Accordingly, the secondary path $S(z)$, comprising the digital-to-analog converter (DAC), reconstruction filter, power amplifier, loudspeaker, acoustic path, error microphone, preamplifier, antialiasing filter, and analog-to-digital converter (ADC) in practice, is assumed to be time-invariant but unknown [2]. The white noise $x_{w h}(n)$ is considered to be a common source of stimulation signals in the estimation of $S(z)$. Figure 1 presents the offline model, in which the error signal $e_{w h}(n)$ is picked up by the error sensor and is determined as

$$
e_{w h}(n)=x_{w h}^{\prime}(n)-\widehat{x}_{w h}^{\prime}(n),
$$

where $\widehat{x}_{w h}^{\prime}(n)$ is the output of the adaptive filter and is represented as

$$
\widehat{x}_{w h}^{\prime}(n)=\sum_{l=0}^{L-1} \widehat{s}_{l}(n) x_{w h}(n-l)=\widehat{s}^{T}(n) \mathbf{x}_{w h}(n),
$$

where $\quad \mathbf{x}_{w h}(n)$ is determined by vector $\mathbf{x}_{w h}(n) \equiv\left[x_{w h}(n), x_{w h}(n-1), \ldots, x_{w h}(n-L+1)\right]^{T} \quad$ and emitted by the secondary loudspeaker at time $n$ and $\widehat{s}_{l}(n)$ is the $l^{\text {th }}$ adaptive coefficient of the finite impulse response (FIR) filter $\widehat{S}(z)$, with $\widehat{s}(n) \equiv\left[\widehat{s}_{0}(n), \widehat{s}_{1}(n), \ldots, \widehat{s}_{L-1}(n)\right]^{T}$. The LMS algorithm updates the coefficients for the adaptive filter as

$$
\widehat{\boldsymbol{s}}(n+1)=\widehat{\boldsymbol{s}}(n)+\mu \mathbf{x}_{w h}(n) e_{w h}(n) .
$$

Applying the convergence conditions of the mean square error (MSE) [39] yields step size $\mu$ that satisfies the boundary condition

$$
0<\mu<\frac{2}{3 L P_{x}(n)},
$$

where $P_{x}(n)$ denotes the power of input signal $x_{w h}(n)$.

\subsection{Conventional Methods for Reducing Narrowband Noise}

2.2.1. Narrowband Feedforward ANC Method. Figure 2 displays the parallel form NANC model. This is the typical model for the conventional narrowband feedforward ANC (CNFANC) method. $P(z)$ is the primary path, and $d(n)$ is the primary noise comprising $k$ harmonics and is assumed to be 


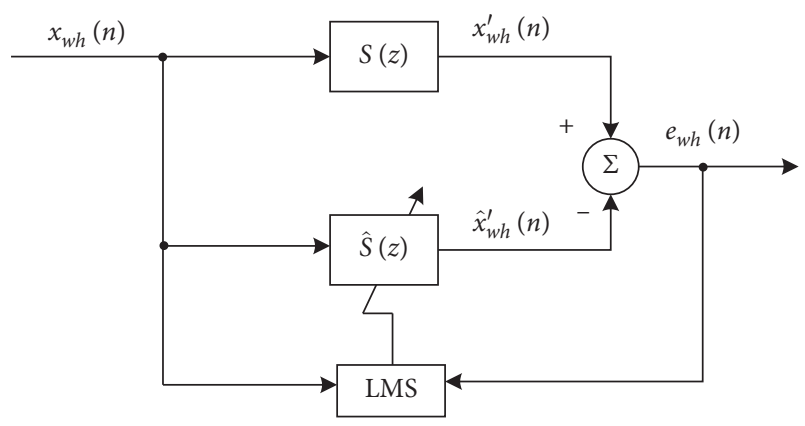

Figure 1: The offline model.

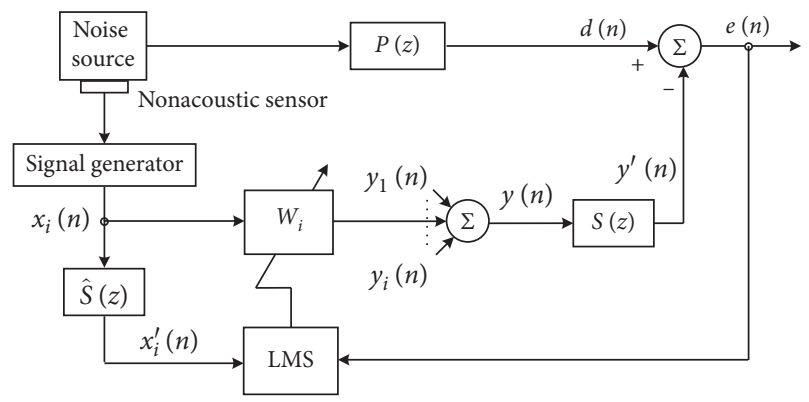

Figure 2: Conventional parallel form NANC model.

$$
\mathrm{d}(n)=\sum_{i=1}^{k} d_{i}(n)
$$

where $\mathrm{d}_{i}(n)$ is the undesired narrowband noise with frequency $\omega_{i}$. The reference input at the $i^{\text {th }}$ channel of the NANC system is a sinusoidal signal

$$
x_{i}(n)=\cos \left(\omega_{i} n\right),
$$

with the angular frequency $\omega_{i}$. The reference input signal is usually produced from the information provided by the synchronization signal from a nonacoustic sensor to the noise source. The output signal of adaptive filter at the $i^{\text {th }}$ channel is

$$
y_{i}(n)=\sum_{l=0}^{L-1} w_{i}(n) x_{i}(n-l)=\mathbf{w}_{i}^{T}(n) \mathbf{x}_{i}^{w}(n),
$$

where $\quad \mathbf{x}_{i}^{w}(n)=\left[x_{i}(n), x_{i}(n-1), \ldots, x_{i}(n-L+1)\right]^{T}$, $\mathbf{w}_{i}(n)=\left[w_{i, 0}(n), w_{i, 1}(n), \ldots, w_{i, L-1}(n)\right]^{T}$, and $L$ is the filter length of $W_{i}(z)$. The antinoise signal $y(n)$ is the sum of each channel, and we have

$$
y(n)=\sum_{i=1}^{k} y_{i}(n)
$$

and the error signal

$$
e(n)=d(n)-y^{\prime}(n) .
$$

The filtered signal is

$$
\mathrm{x}_{i}^{\prime}(n)=\sum_{m=0}^{M-1} \widehat{s}(n) x_{i}^{\prime}(n-m)=\widehat{s}^{T}(n) \mathbf{x}_{i}^{\prime}{ }^{\prime}(n),
$$

where $\widehat{s}(n)=\left[\widehat{\boldsymbol{s}}_{0}(n), \widehat{\boldsymbol{s}}_{1}(n), \ldots, \widehat{\boldsymbol{s}}_{M-1}(n)\right]^{T}$ is the vector coefficient of the estimated secondary path $\widehat{S}(z)$ and $\mathbf{x}_{i}^{\prime}(n)=\left[x_{i}(n), x_{i}(n-1), \ldots, x_{i}(n-M+1)\right]^{T}$. By setting the cost function as

$$
\xi(n)=E\left[e^{2}(n)\right],
$$

we can obtain the estimated gradient

$$
\nabla \xi(n)=\frac{\partial \xi(n)}{\partial \mathbf{w}_{i}(n)}=-2 e(n) \mathbf{x}_{i}^{\prime}(n)
$$

The FXLMS algorithm for updating the weights is

$$
\begin{aligned}
\mathbf{w}_{i}(n+1) & =\mathbf{w}_{i}(n)-\frac{\mu_{\text {conv }} \nabla \xi(n)}{2}, \\
& =\mathbf{w}_{i}(n)+\mu_{\text {conv }} e(n) \mathbf{x}_{i}^{\prime}(n),
\end{aligned}
$$

where $\mu_{\text {conv }}$ is the step size and $\mathbf{x}_{i}^{\prime}(n)=\left[x_{i}^{\prime}(n)\right.$, $\left.x_{i}^{\prime}(n-1), \ldots, x_{i}^{\prime}(n-L+1)\right]$ is the filtered signal vector.

2.2.2. Feedback ANC Method. The feedback-based ANC model is shown in Figure 3. By only using the error sensor to measure the residual noise signal $e(n)$, the adaptive feedback ANC algorithm synthesizes the reference signal $x(n)$ as

$$
x(n) \equiv \widehat{d}(n)=e(n)+\sum_{m=0}^{M-1} \widehat{s}_{m} y(n-m),
$$

where $\widehat{s}_{m}(m=0,1, \ldots, M-1)$ is the coefficients of the filter $\widehat{S}(z)$. Thus, we have

$$
v(n)=\sum_{m=0}^{M-1} \widehat{s}_{m} y(n-m) .
$$

The antinoise signal $y(n)$ is generated as

$$
y(n)=\sum_{l=0}^{l-1} w_{l}(n) x(n-l)
$$

where $w_{l}(n)(l=0,1, \ldots, L-1)$ is the coefficients of $W(z)$ at time $n$ and $L$ is the filter length of $W(z)$. The FXLMS algorithm updates the weights of adaptive FIR filter and is given by

$$
\mathbf{w}_{l}(n+1)=\mathbf{w}_{l}(n)+\mu \widehat{d}^{\prime}(n-l) e(n),
$$

where $\mu$ is the step size. The filtered reference signal is defined as

$$
\widehat{\mathrm{d}} \prime(n) \equiv \sum_{m=0}^{M-1} \widehat{s}_{m} \widehat{\mathrm{d}}(n-m)
$$

2.2.3. Adaptive Neural Controller. Based on the feedback ANC system, the adaptive neural network approach including input layer, hidden layer, and output layer was presented in [35]. Two adaptive algorithms determine the weights for the hidden and the output layers. Figure 4 shows the structure suggested in [35]. 


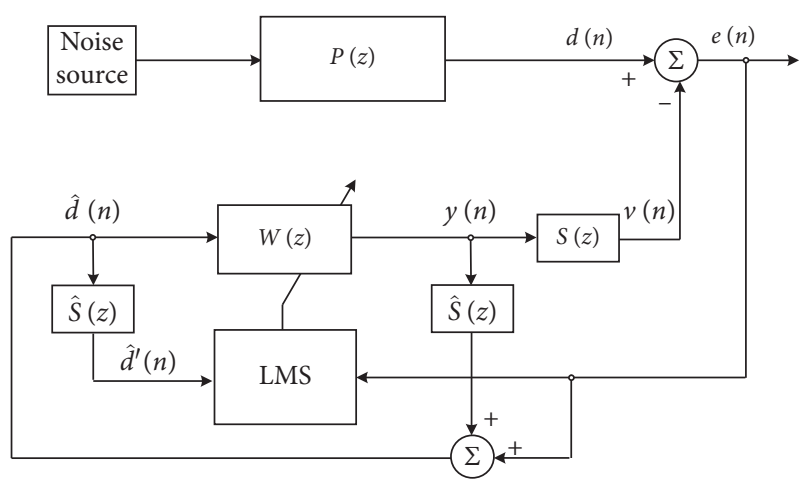

Figure 3: Narrowband feedback ANC model.

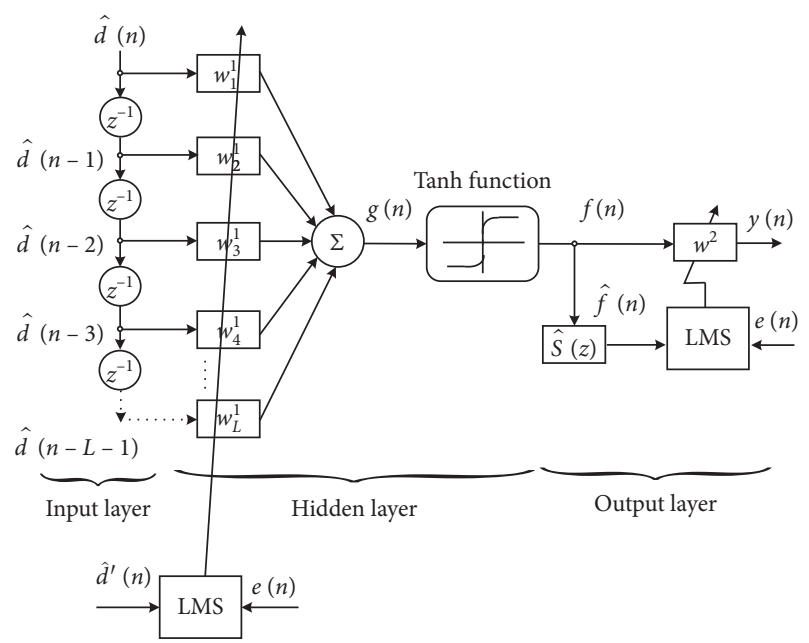

FIGURE 4: Structure of previous controller in [35].

$f(n)$ is the output signal of the hidden layer with the activation function Tanh (), and $y(n)$ represents the output of the output layer, indicating the antinoise signal. The signal $e(n)$ denotes the error signal.

\section{The Proposed Method and Mathematical Analysis}

This section analyzes the NAFNC algorithm and considers its convergence and stability properties.

3.1. Algorithm of the Proposed Method. Firstly, using the parallel NANC structure, the proposed method is presented in Figure 5. The primary noise $d(n)$ comprises $k$ harmonics, and $x_{i}(n)$ is the $i^{\text {th }}$ reference sinusoidal signal.

The error signal is given by the equation:

$$
e(n)=\mathrm{d}(n)-y^{\prime}(n),
$$

where $y^{\prime}(n)=s(n) * y(n)$, with “ * " denoting linear convolution operation and $s(n)$ being the impulse response of $S(z), y(n)$ is the sum of the antinoise signal of the NAFNC:

$$
y(n)=\sum_{i=1}^{k} y_{i}(n)
$$

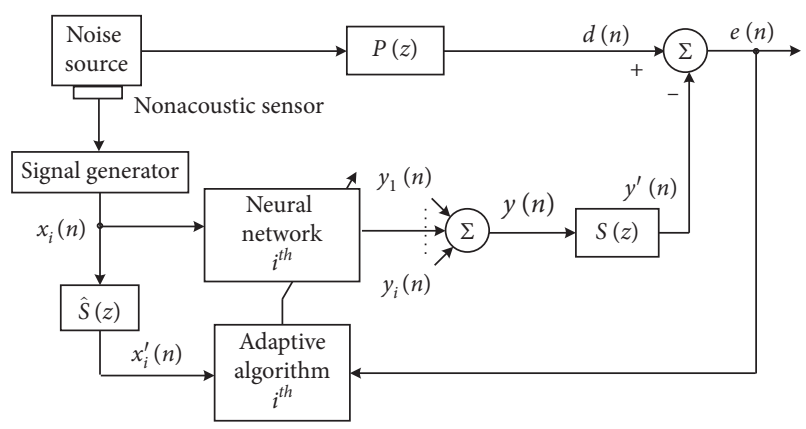

FIgURE 5: The proposed method.

Figure 6 displays the structure of the proposed NAFNC. Here, a three-layer perceptron feedback neural network is constructed, and the FXLMS algorithm is used to update the adaptive weights $\mathbf{w}_{1 i}(n)$ and $\mathbf{w}_{2 i}(n)$. Layer 1 is the input layer that directly receives the input signal and transmits the signal to the hidden layer. Layer 2 is the hidden layer whose output signal has the sigmoid activation function. The feedback component is connected from the output of layer 2 to its input through weights $w_{1 i}(n)$ and the adjustment parameter $\alpha$. Layer 3 is the output layer. The algorithm of the NAFNC is as follows:

Layer 1:

$$
\operatorname{layer}_{i}^{1}(n)=x_{i}(n), \quad i=1,2,3, \ldots, k
$$

Layer 2:

$$
\operatorname{layer}_{i}^{2}(n)=g_{i}\left(q_{i}(n)\right) \text {, }
$$

with $q_{i}(n)=\sum_{l=0}^{L-1}\left(w_{1 i}(n) x_{i}(n-l)+\alpha w_{1 i}(n) g_{i}(n-l-1)+B\right)$.

And the activation function at the $i^{\text {th }}$ channel is defined as

$$
g_{i}(n)=\operatorname{sigmoid}\left(q_{i}(n)\right)=\frac{1}{1+e^{-q_{i}(n)}}
$$

where $B$ is the bias parameter and $\alpha$ is the adjustment parameter that adjusts the feedback signal of the hidden layer so that the system achieves a balanced output signal. The cost function is determined as $\xi(n)=E\left[e^{2}(n)\right]$. The gradient estimation of layer 2 at time $n$ is

$$
\begin{aligned}
\nabla \xi_{1 i}(n)= & \frac{\partial \xi_{1 i}}{\partial w_{1 i}}=-2 e(n) \sum_{m=0}^{M-1} s_{m} x_{i}(n-m) \mathbf{w}_{2 i} \\
& \cdot(n) g_{i}(n-m)\left(1-g_{i}(n-m)\right),
\end{aligned}
$$

where $\mathbf{w}_{2 i}(n)$ is the weight of layer 3 and $\mathbf{w}_{1 i}(n)$ is the weight of layer 2 updated by the FXLMS algorithm:

$$
\begin{aligned}
& \mathbf{w}_{1 i}(n+1)=\mathbf{w}_{1 i}(n)-\frac{\mu_{1 \_ \text {prop }}}{2} \nabla \xi_{1 i}(n), \\
& \text { with } \mathrm{x}_{i}^{\prime}(n)=\sum_{m=0}^{M-1} \widehat{s}_{m}(n) x_{i}(n-m),
\end{aligned}
$$




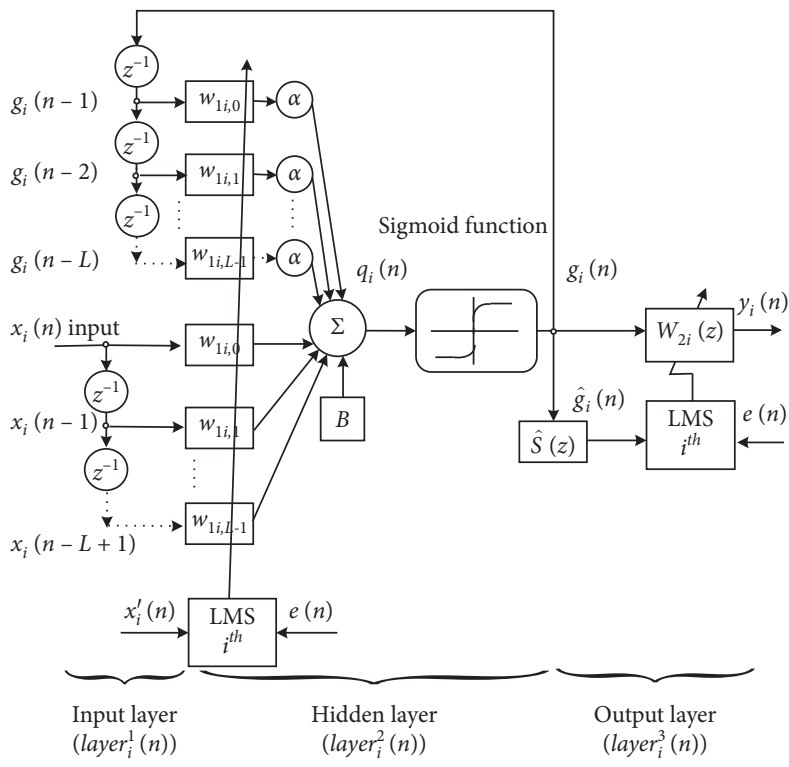

FIgURE 6: Structure of the presented NAFNC.

where $\widehat{s}_{m}(n),(m=0, \ldots, M-1)$, are the coefficients of $\widehat{S}(z)$.

Substituting equation (25) and equation (27) into equation (26) yields

$\mathbf{w}_{1 i}(n+1)=\mathbf{w}_{1 i}(n)+\mu_{1 \_ \text {prop }} \mathbf{x}_{\mathbf{i}}^{\prime}(n) e(n) \mathbf{w}_{2 i}(n) g_{i}(n)\left(1-g_{i}(n)\right)$,

where $\mathbf{x}_{i}^{\prime}(n)=\left[x_{i}(n), x_{i}(n-1), \ldots, x_{i}(n-L+1)\right]^{T}$ is the filtered signal vector; $\quad \mathbf{w}_{1 i}(n) \equiv\left[w_{1 i, 0}(n) w_{1 i, 1}\right.$ $\left.(n) w_{1 i, 2}(n), \ldots, w_{1 i, L-1}(n)\right]^{T}$ is the weight vector of layer 2 ; $e(n)$ is the error signal; $\mu_{1-\text { prop }}$ is the learning rate parameter or the step size of layer 2 .

The output of layer 3 is

$$
\operatorname{layer}_{i}^{3}(n)=y_{i}(n)=\sum_{l=0}^{L-1} w_{2 i}(n) g_{i}(n-l)=\mathbf{w}_{2 i}^{T}(n) \mathbf{g}_{i}^{w}(n),
$$

where $\mathbf{w}_{2 i}(n) \equiv\left[w_{2 i, 0}(n), w_{2 i, 1}(n), w_{2 i, 2}(n), \ldots, w_{2 i, L-1}(n)\right]^{T}$ is the weight vector of $W_{2 i}(z)$ and the filtered signal vector $\mathbf{g}_{i}^{w}(n)=\left[g_{i}(n), g_{i}(n-1), \ldots, g_{i}(n-L+1)\right]^{T}$.

With $\widehat{g}_{i}(n)=\sum_{m=0}^{M-1} \widehat{s}_{m}(n) g_{i}(n-m)=\widehat{s}^{T}(n) \mathbf{g}_{i}^{s}(n)$, $\widehat{s}(n)=\left[\widehat{s}_{0}(n), \widehat{s}_{1}(n), \ldots, \widehat{s}_{M-1}(n)\right]^{T}$ is the filtered vector and $\mathbf{g}_{i}^{s}(n)=\left[g_{i}(n), g_{i}(n-1), \ldots, g_{i}(n-M+1)\right]^{T}$. Similarly, the estimated gradient to tune the weight vector of layer 3 at time $n$ is

$$
\nabla \xi_{2 i}(n)=\frac{\partial \xi_{2 i}}{\partial w_{2 i}}=-2 e(n) \widehat{g}_{i}(n)
$$

Therefore, we have

$$
\begin{aligned}
\mathbf{w}_{2 i}(n+1) & =\mathbf{w}_{2 i}(n)-\frac{\mu_{2}{ }_{-p r o p}}{2} \nabla \xi_{2 i}(n) \\
& =\mathbf{w}_{2 i}(n)+\mu_{2 \text { _prop }} e(n) \widehat{g}_{i}(n),
\end{aligned}
$$

where $\mu_{2}$ prop is the step size, $\widehat{g}_{i}(n)=\left[g_{i}(n), g_{i}(n-1), \ldots, g_{i}(n-L+1)\right]^{T}$ is the filtered signal vector $(i=1,2,3, \ldots, k)$, and $e(n)$ is the error signal at time $n$.

Comparing Figures 6 and 4 indicates that the proposed method uses a feedback component that is combined with adaptive weights $\mathbf{w}_{1 i}(n)$ through the adjustment parameter $\alpha$ and the bias parameter $B$. Based on the FXLMS algorithm, two adaptive algorithms tune the weight vectors of the hidden and the output layers, which helps the presented system achieve rapid convergence without any prior training of neural networks. Both the adjustment parameter $\alpha$ and bias parameter $B$ are determined experimentally to achieve convergence and stability of the system.

3.2. Convergence and Stability of the Proposed Method. The NAFNC is designed with two FXLMS algorithms for rapid and stable convergence. The weight vectors $\mathbf{w}_{1 i}(n)$ and $\mathbf{w}_{2 i}(n)$ are updated online to minimize the error signal. In order to verify the convergence and stability of the proposed work, the Lyapunov function is chosen as

$$
C(n)=\frac{1}{2} e^{2}(n)=\frac{1}{2}\left[\mathrm{~d}(n)-y^{\prime}(n)\right]^{2} .
$$

Therefore, $\Delta C(n)$ can be calculated as follows:

$$
\begin{aligned}
\Delta C(n) & =C(n+1)-C(n)=\frac{\left[e^{2}(n+1)-e^{2}(n)\right]}{2} \\
& =\frac{[e(n)+e(n+1)][e(n+1)-e(n)]}{2} \\
& =\frac{\Delta e(n)[\Delta e(n)+2 e(n)]}{2},
\end{aligned}
$$

where $\Delta e(n)$ is used to update the weights $\mathbf{w}_{2 i}(n)$ of the output layer computed as 


$$
\begin{aligned}
\Delta e(n) & =e(n+1)-e(n)=\left[\frac{\partial e}{\partial w_{2 i}}\right] \Delta \mathbf{w}_{2 i}(n) \\
& =\left[\frac{\partial e(n)}{\partial y^{\prime}(n)} \sum_{m=0}^{M-1} \frac{\partial y^{\prime}(n)}{\partial y_{i}(n-m)} \frac{\partial y_{i}(n-m)}{\partial \mathrm{w}_{2 i}(n)}\right] \Delta \mathbf{w}_{2 i}(n) \\
& =\left\{-\sum_{m=0}^{M-1} s_{m}(n) g_{i}(n-m)\right\}\left\{\mu_{2 \text { _prop }} e(n) \sum_{m=0}^{M-1} s_{m}(n) g_{i}(n-m)\right\} \\
& =-\mu_{2 \_ \text {prop }} e(n)\left\|\sum_{m=0}^{M-1} s_{m}(n) g_{i}(n-m)\right\|^{2} .
\end{aligned}
$$

Substituting equation (34) into equation (33) yields

$$
\begin{aligned}
\Delta C(n)= & -\frac{1}{2} \mu_{2 \text { prop }} e(n)\left\|\sum_{m=0}^{M-1} s_{m}(n) g_{i}(n-m)\right\|^{2} \\
& \times\left[2 e(n)-\mu_{2 \text { prop }} e(n)\left\|\sum_{m=0}^{M-1} s_{m}(n) g_{i}(n-m)\right\|^{2}\right] \\
= & -\frac{1}{2} \mu_{2 \text { prop }} e^{2}(n)\left\|\sum_{m=0}^{M-1} s_{m}(n) g_{i}(n-m)\right\|^{2} \\
& \times\left[2-\mu_{2} \text { prop }\left\|\sum_{m=0}^{M-1} s_{m}(n) g_{i}(n-m)\right\|^{2}\right] .
\end{aligned}
$$

From equation (35), it is obvious that if the learning rate $\mu_{2 \text { _prop }}$ is chosen to satisfy

$$
0<\mu_{2-\text { prop }}<\frac{2}{\left\|\sum_{m=0}^{M-1} s_{m}(n) g_{i}(n-m)\right\|^{2}}
$$

then the stability of the system is ensured. The proof to derive the boundary of $\mu_{1 \text { _prop }}$ is the same as equations (35) and (36); therefore, the weight $\mathbf{w}_{1 i}(n)$ converges by setting $\mu_{1 \_ \text {prop }}$ as

$$
0<\mu_{1} \text { prop }<\frac{2}{\left\|\sum_{m=0}^{M-1} s_{m} x_{i}(n-m) w_{2 i}(n) g_{i}(n-m)\left(1-g_{i}(n-m)\right)\right\|^{2}}
$$

Equations (36) and (37) specify convergence and stability conditions of NAFNC, depending on step sizes $\mu_{1}$ prop

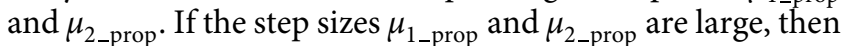
the convergence speed will be large accordingly. But if the step size $\mu_{\text {prop }}$ violates the boundary condition, then the system will become unstable. Therefore, the appropriate value of the step sizes $\mu_{1 \text { _prop }}$ and $\mu_{2 \text { _prop }}$ must be chosen experimentally to ensure good performance.

\section{Simulation Results}

Simulations are used to compare the performance of the proposed work with the previously presented method in [35]. Thereby, the superiority of the proposed method will be discussed. The sampling frequency is $4 \mathrm{kHz}$ for the initial setting of the general system parameter.

4.1. Case 1 . The primary path $P(z)$ and the secondary path $S(z)$ of the ANC system in case 1 are estimated from [2] with the length 256 , and $\widehat{S}(z)$ is set the same as $S(z)$. The noise source is the synthesis of the narrowband noise, considering only the three components $(i=1,2,3)$ with amplitude 1 at $100 \mathrm{~Hz}, 200 \mathrm{~Hz}$, and $300 \mathrm{~Hz}$ and being combined with a white noise signal with amplitude of 0.0049 . The length of the adaptive FIR filter of the CNFANC method is 150 . The order of the adaptive filter of the hidden layer and the output layer of the previous method in [35] and of the proposed method is 150. Regarding these parameters for case 1, the step size of the CNFANC method is $\mu_{\text {conv }}=0.8 \times 10^{-4}$ and of the proposed method is $\mu_{1 \text { _prop }}=0.2$ and $\mu_{2 \text { _prop }}=2.1 \times 10^{-3}$. The bias parameter is $B=0.01$ and the adjustment parameter is $\alpha=0.041$. The experiment of case 1 is performed to adjust the parameters by trial and error method to assure the system convergence and stability. The parameters for [35] include the step size of the hidden layer ( $\left.\mu_{1_{-} \text {prev }}\right)$ being 0.2 and the step size of the output layer $\left(\mu_{2}\right.$ prev $)$ being $1.1 \times 10^{-4}$. Figure 7 plots the error signals in the frequency domain, including the CNFANC method (blue), the previous method in [35] (green), the proposed method (red), and the noise signal $d(n)$ (gold). The CNFANC method and 


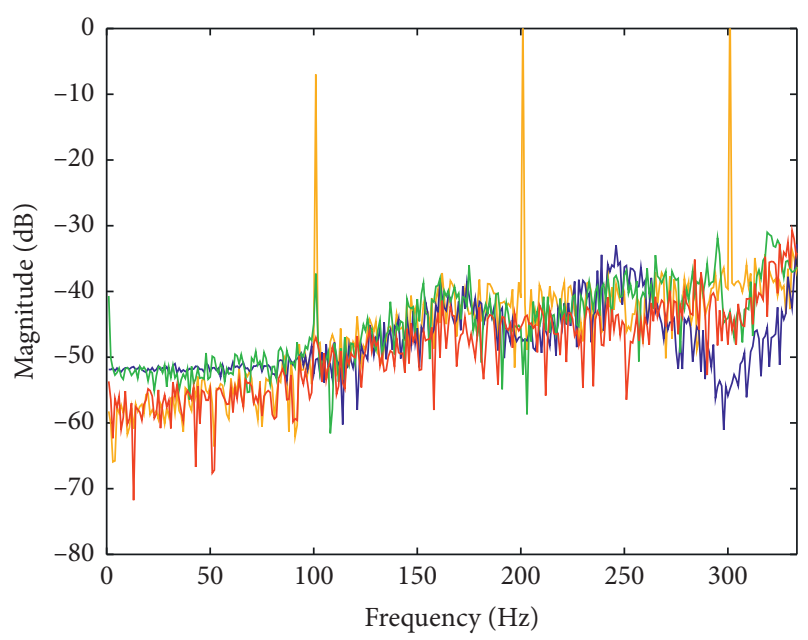

FIGURE 7: Error and noise signals in frequency domain, showing the error signals in the CNFANC method (blue), the previous method in [35] (green), and the proposed method (red), as well as the noise signal $d(n)$ (gold).

the proposed method thoroughly eliminate the noise with all frequencies, whereas the previous method in [35] eliminates the noise at only $200 \mathrm{~Hz}$ and $300 \mathrm{~Hz}$. Figure 8 plots the learning curve. Obviously, all three methods perform equally in terms of the noise reduction performance. However, the convergence speed of the proposed method and the CNFANC method is better than the existing method in [35].

4.2. Case 2. In case 2 , the secondary path remains as in case 1 , but the nonlinear primary path is considered [35].

$$
\mathrm{d}(n)=x(n-3)-0.3 x(n-4)+0.2 x(n-5)+0.8 x^{2}(n-5),
$$

where $x(n)$ contained sinusoidal signal components at $100 \mathrm{~Hz}, 200 \mathrm{~Hz}$, and $300 \mathrm{~Hz}$ with amplitude 1 and was combined with the white noise signal with amplitude of 0.0049. The order of adaptive filter in the CNFANC method, the previously presented method in [35], and the proposed method is selected the same as case 1. The step size for the CNFANC method is $\mu_{\text {conv }}=0.06 \times 10^{-5}$. The parameters $\mu_{1 \_ \text {prop }}=0.5$ and $\mu_{2 \text { _prop }}=3.4 \times 10^{-4}$, the bias parameter $B=0.021$, and the adjustment parameter $\alpha=0.2$ were used for the proposed work. The step sizes for [35] are $\mu_{1-\text { prev }}=$ 0.21 and $\mu_{2 \text { _prev }}=6.4 \times 10^{-5}$. Figure 9 shows the noise and error signals in time domain. The proposed method is most efficient in reducing the noise. Figure 10 plots the learning curve. The proposed method is effective in reducing narrowband noise but the other methods failed to reduce the noise efficiently.

4.3. Case 3. Case 3 considers the ANC system involving both nonlinear primary and secondary paths. The frequencies of the undesired narrowband noises are changed abruptly to see the responsiveness of the proposed method too. The nonlinear primary path has been modelled as a cascade of a linear filter and a nonlinear one. The former is defined as

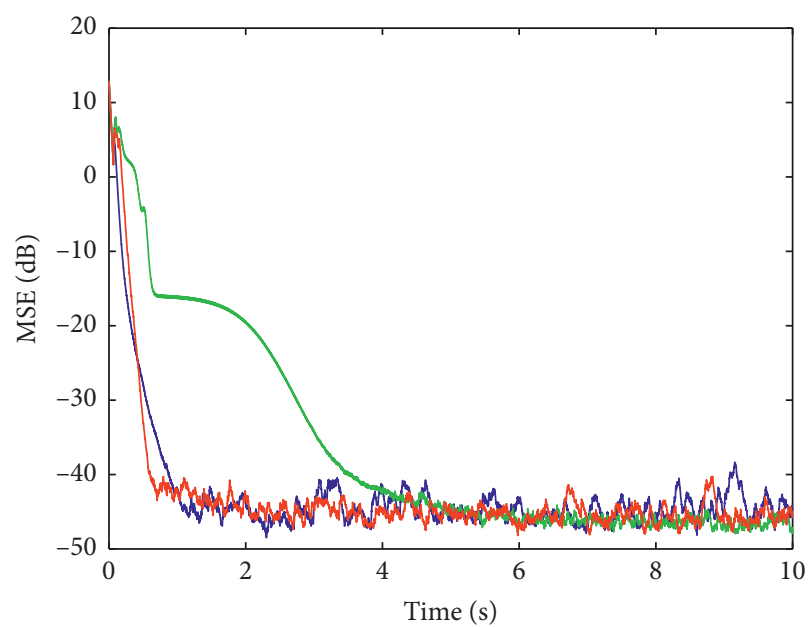

FIgURE 8: Learning curve of MSE for the CNFANC method (blue), the previous method in [35] (green), and the proposed method (red).

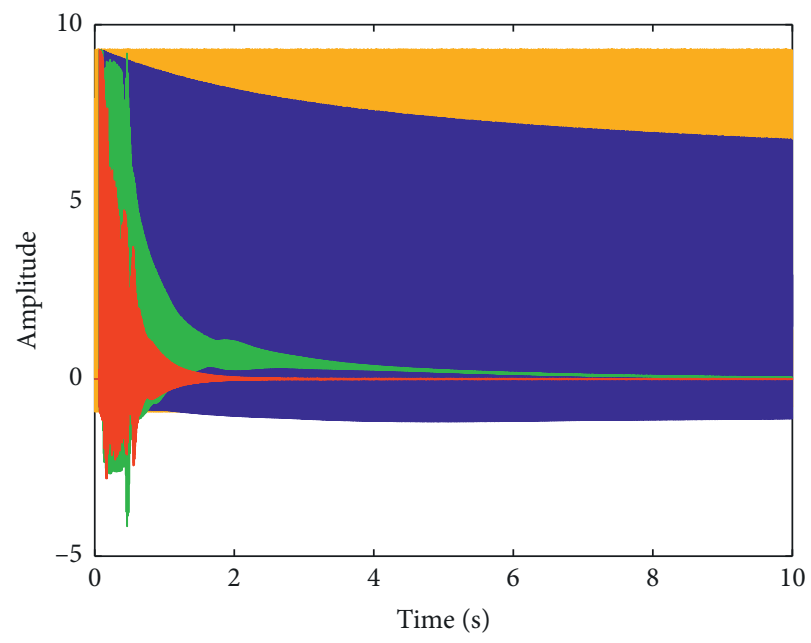

FIGURE 9: Noise and error signals in time domain, error signals in the CNFANC method (blue), the previous method in [35] (green), and the proposed method (red), as well as the noise signal $d(n)$ (gold).

$t(n)=x(n-2)-0.9 x(n-3)+0.01 x(n-5)$, and the latter is selected based on a third-order polynomial model [40-42]: $\mathrm{d}(n)=t(n-2)+0.08[t(n-2)]^{2}+0.04[t(n-2)]^{3}$, and the nonlinear secondary path is given by a Volterra series [43] $y^{\prime}(n)=y(n)+0.35 y(n-1)+0.09 y(n-2)-0.5 y(n) y$ $(n-1)+0.4 y(n) y(n-2)$, according to Figure 5 . The frequencies of the three narrowband components $(i=1,2,3)$ are $100 \mathrm{~Hz}, 200 \mathrm{~Hz}$, and $400 \mathrm{~Hz}$ at first and suddenly changed to $150 \mathrm{~Hz}, 250 \mathrm{~Hz}$, and $300 \mathrm{~Hz}$ at the $5^{\text {th }}$ second. Each narrowband noise is with amplitude 1, combining with the white noise with amplitude 0.1 . The order of adaptive filter in the CNFANC method, the previous method in [35], and the proposed method is increased to 200 . The step size for the CNFANC method is $\mu_{\text {conv }}=0.02 \times 10^{-4}$ and the parameters are $\mu_{1_{\text {_prop }}}=0.8, \mu_{2_{\text {_prop }}}=4 \times 10^{-4}, B=0.1 \times 10^{-2}$, and $\alpha=$ $0.1 \times 10^{-3}$ for the proposed method. The step sizes for [35] 


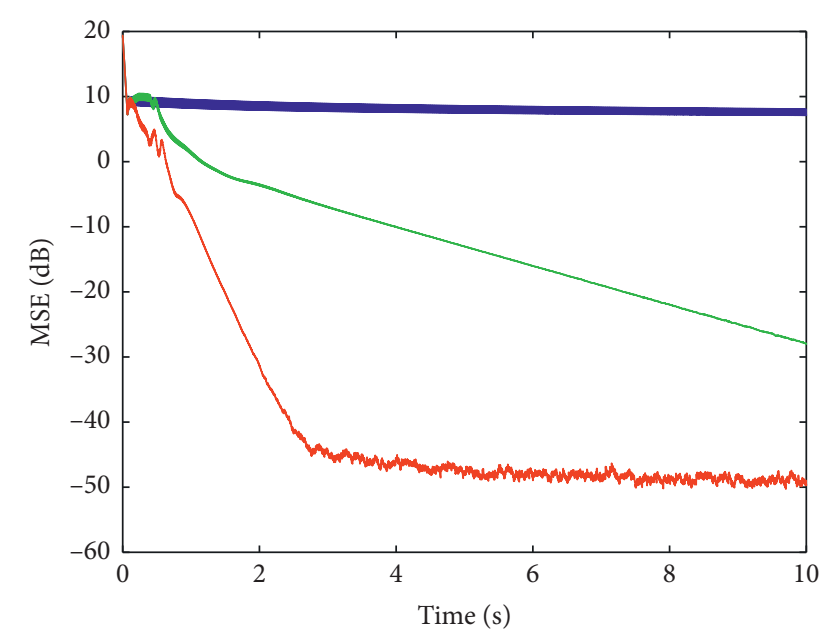

FIGURE 10: Learning curve of MSE for the CNFANC method (blue), the previous method in [35] (green), and the proposed method (red).

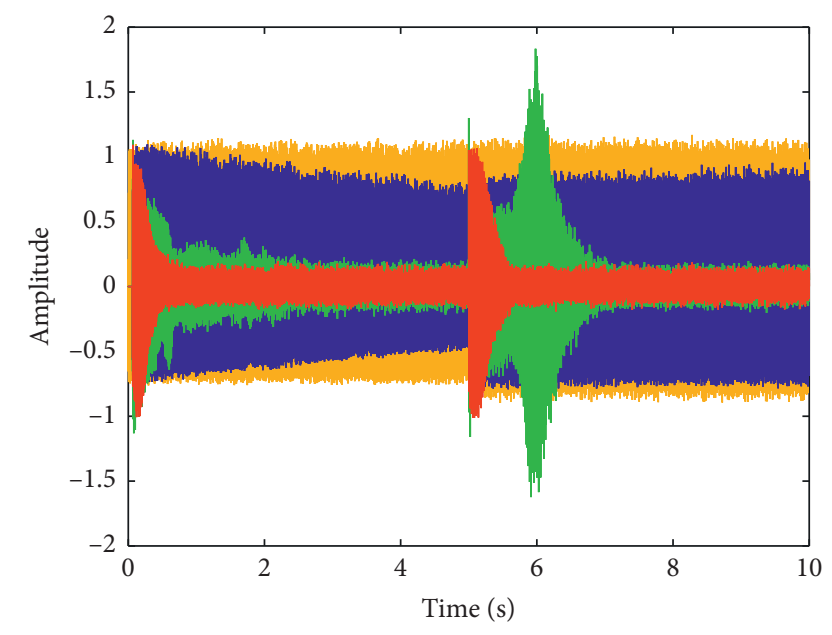

FIgURE 11: Noise and error signals in time domain, error signals obtained using the CNFANC method (blue), the previous method in [35] (green), and the proposed method (red), as well as the noise signal $d(n)$ (gold).

are $\mu_{1 \text { _prev }}=0.8$ and $\mu_{2 \text { _prev }}=0.1 \times 10^{-2}$. Figure 11 presents the error signals in time domain. The convergence rate of the proposed method obviously outperforms those of the other schemes. Although frequencies change abruptly at the $5^{\text {th }}$ second, the proposed method is still stable and converges quickly. Figure 12 plots the learning curves. The results show that the proposed method is still good at reducing the noise in nonlinear system.

These experiments confirm the noise reduction efficiency of the proposed method. The proposed method is effective not only for linear ANC systems but also for nonlinear ANC systems. In contrast, the CNFANC method is effective only for linear ANC systems, as in case 1, but not for nonlinear ANC systems as in cases 2 and 3. Moreover, the proposed method offers improved convergence speed and performance owing to the use of the adjustment parameter $\alpha$ and

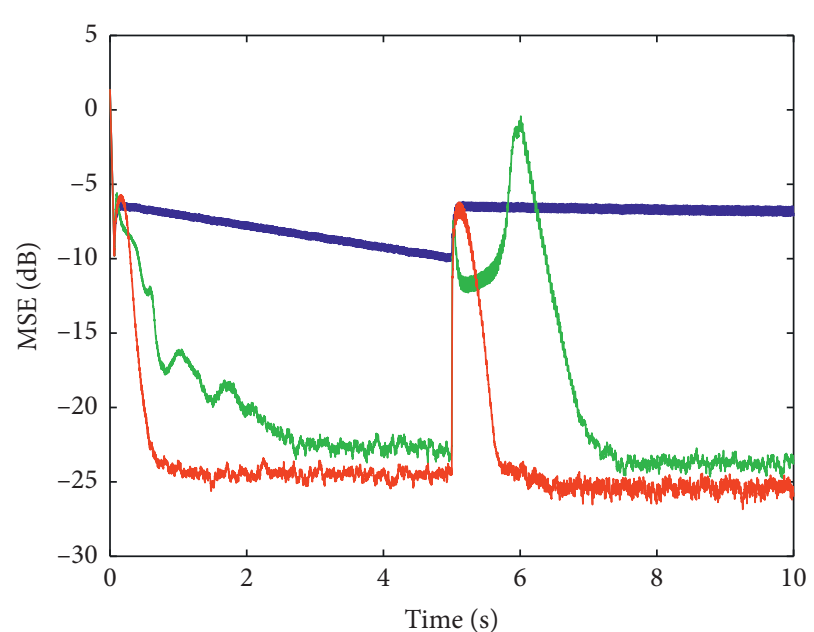

FIGURE 12: Learning curves of the MSE for the CNFANC method (blue), the previous method in [35] (green), and the proposed method (red).

bias parameter $B$ in various ANC systems. The adjustment parameter $\alpha$ is effectively combined with the bias parameters $B$ according to equation (23) so that the step sizes $\mu_{1 \text { _prop }}$ and $\mu_{2}$ prop can reach high values matching the boundary conditions from equations (36) and (37), making the proposed method converge quickly, as proven by the experimental results. However, adding a feedback component to the hidden layer increases the cost of calculation and could make the system unstable. Hence, the parameter $\alpha$ must be adjusted carefully to enable the ANC system achieving stable and quick convergence.

\section{Conclusions}

This paper analyzed the NAFNC algorithm and considered its stability and convergence. Simulation results confirmed the superiority of the proposed method based on three simulations. Although the proposed method costed computation, it performed well for both linear and nonlinear conditions. In addition, the advantages of the proposed method included the fast and stable convergence. The addition of the feedback element in the hidden layer in the proposed method improved the system performance.

\section{Data Availability}

The data used to support the findings of this study are available within the article.

\section{Conflicts of Interest}

The authors declare that they have no conflicts of interest.

\section{References}

[1] L. Paul, Process of Silencing Sound Oscillations, United State Patent Office, Alexandria, VA, USA, 1936.

[2] S. M. Kuo and D. R. Morgan, Active Noise Control Systems: Algorithms and DSP Implementation, Wiley, New York, NY, USA, 1996. 
[3] G. Gäbel, J. Millitzer, H. Atzrodt, S. Herold, and A. Mohr, "Development and implementation of a multi-channel active control system for the reduction of road induced vehicle interior noise," Actuators, vol. 7, no. 3, p. 52, 2018.

[4] Y. S. Wang, H. Guo, Y. R. Li, N. N. Liu, and C. Yang, "Active control for vehicle interior noise based on DWT-FxLMS algorithm using a piezoelectric feedback system," Applied Acoustics, vol. 167, Article ID 107409, 2020.

[5] H. Guo, Y. S. Wang, C. Yang, X. L. Wang, N. N. Liu, and Z. J. Xu, "Vehicle interior noise active control based on piezoelectric ceramic materials and improved fuzzy control algorithm," Applied Acoustics, vol. 150, pp. 216-226, 2019.

[6] H. Guo, Y. S. Wang, N. N. Liu, R. P. Yu, H. Chen, and $\mathrm{X}$. T. Liu, "Active interior noise control for rail vehicle using a variable step-size median-LMS algorithm," Mechanical Systems and Signal Processing, vol. 109, pp. 15-26, 2018.

[7] J. Zeng and R. De Callafon, "Recursive filter estimation for feedforward noise cancellation with acoustic coupling," Journal of Sound and Vibration, vol. 291, no. 3-5, pp. 10611079, 2006.

[8] P. Peretti, S. Cecchi, L. Romoli, and F. Piazza, "Adaptive feedback active noise control for yacht environments," Institute of Electrical and Electronics Engineers Transactions on Control Systems Technology, vol. 22, no. 2, pp. 737-744, 2013.

[9] L. Wu, X. Qiu, and Y. Guo, "A simplified adaptive feedback active noise control system," Applied Acoustics, vol. 81, pp. 40-46, 2014.

[10] C.-Y. Ho, K.-K. Shyu, C.-Y. Chang, and S. M. Kuo, "Efficient narrowband noise cancellation system using adaptive line enhancer," Institute of Electrical and Electronics Engineers/ ACM Transactions on Audio, Speech, and Language Processing, vol. 28, pp. 1094-1103, 2020.

[11] J. $\mathrm{Hu}$ and T. Hsiao, "Adaptive feedforward active noise cancellation in ducts using the model matching of wave propagation dynamics," Institute of Electrical and Electronics Engineers Transactions on Control Systems Technology, vol. 20, no. 5, pp. 1351-1356, 2011.

[12] B. Huang, Y. Xiao, J. Sun, and G. Wei, "A variable step-size FXLMS algorithm for narrowband active noise control," Institute of Electrical and Electronics Engineers Transactions on Audio, Speech, and Language Processing, vol. 21, no. 2, pp. 301-312, 2012.

[13] C. Y. Chang and S. M. Kuo, "Complete direct/parallel structure for narrowband active noise control systems," IET Signal Processing, vol. 7, no. 6, pp. 477-485, 2013.

[14] C.-Y. Chang and S. M. Kuo, "Complete parallel narrowband active noise control systems," Institute of Electrical and Electronics Engineers Transactions on Audio, Speech, and Language Processing, vol. 21, no. 9, pp. 1979-1986, 2013.

[15] C.-Y. Chang, S. M. Kuo, and C.-W. Huang, "Secondary path modeling for narrowband active noise control systems," Applied Acoustics, vol. 131, pp. 154-164, 2018.

[16] J. Shin, H. J. Baek, B. Y. Park, and J. Cho, "A sequential selection normalized subband Adaptive filter with variable step-size algorithms," Mathematical Problems in Engineering, vol. 2018, Article ID 1941367, 2018.

[17] T. Peng, Q. Zhu, M. O. Tokhi, and Y. Yao, "Fuzzy logic feedforward active noise control with distance ratio and acoustic feedback using Takagi-Sugeon-Kang inference," Journal of Low Frequency Noise, Vibration and Active Control, vol. 39, no. 1, pp. 174-189, 2020.

[18] H. Zhao, X. Zeng, Z. He, and T. Li, “Adaptive RSOV filter using the FELMS algorithm for nonlinear active noise control systems," Mechanical Systems and Signal Processing, vol. 34, no. 1-2, pp. 378-392, 2013.

[19] S. M. Kuo and H.-T. Hsien-Tsai Wu, "Nonlinear adaptive bilinear filters for active noise control systems," Institute of Electrical and Electronics Engineers Transactions on Circuits and Systems I: Regular Papers, vol. 52, no. 3, pp. 617-624, 2005.

[20] L. Tan, C. Dong, and S. Du, "On implementation of adaptive bilinear filters for nonlinear active noise control," Applied Acoustics, vol. 106, pp. 122-128, 2016.

[21] J. M. Sousa, C. A. Silva, and J. M. G. Sá da Costa, "Fuzzy active noise modeling and control," International Journal of Approximate Reasoning, vol. 33, no. 1, pp. 51-70, 2003.

[22] I. Aluclu, A. Dalgic, and Z. F. Toprak, "A fuzzy logic-based model for noise control at industrial workplaces," Applied Ergonomics, vol. 39, no. 3, pp. 368-378, 2008.

[23] C.-Y. Chang, K.-K. Shyu, and T.-N. Chuang, "Active noise controller with fuzzy filtered-U algorithm," Electronics Letters, vol. 37, no. 8, pp. 543-545, 2001.

[24] A. Haseeb, M. Tufail, S. Ahmed, M. Rehan, A. Majid, and W. Ahmed, "A fuzzy logic-based gain scheduling method for online feedback path modeling and neutralization in active noise control systems," Fluctuation and Noise Letters, vol. 19, no. 01, Article ID 2050008, 2020.

[25] P. A. Mastorocostas and J. B. Theocharis, "A recurrent fuzzyneural model for dynamic system identification," Institute of Electrical and Electronics Engineers Transactions on Systems, Man and Cybernetics, Part B (Cybernetics), vol. 32, no. 2, pp. 176-190, 2002.

[26] Q.-Z. Zhang, W.-S. Gan, and Y.-L. Zhou, “Adaptive recurrent fuzzy neural networks for active noise control," Journal of Sound and Vibration, vol. 296, no. 4-5, pp. 935-948, 2006.

[27] M. Rakhshan, S. Khorshidi, and B. Safarinejadian, "Active noise control in presence of disturbance using adaptive neuro fuzzy inference system," Journal of Computational Intelligence and Electronic Systems, vol. 3, no. 2, pp. 99-105, 2014.

[28] K. T. Chen, C. H. Chou, S. H. Chang, and Y. H. Liu, "Adaptive fuzzy neural network control on the acoustic field in a duct," Applied Acoustics, vol. 69, no. 6, pp. 558-565, 2008.

[29] J.-W. Park, G. K. Venayagamoorthy, and R. G. Harley, "MLP/ RBF neural-networks-based online global model identification of synchronous generator," Institute of Electrical and Electronics Engineers Transactions on Industrial Electronics, vol. 52, no. 6, pp. 1685-1695, 2005.

[30] T. Krukowicz, "Active noise control algorithm based on a neural network and nonlinear input-output system identification model," Archives of Acoustics, vol. 35, no. 2, pp. 191-202, 2010.

[31] H. Zhao, X. Zeng, and J. Zhang, "Adaptive reduced feedback FLNN filter for active control of nonlinear noise processes," Signal Processing, vol. 90, no. 3, pp. 834-847, 2010.

[32] D. C. Le, J. Zhang, and Y. Pang, "A bilinear functional link artificial neural network filter for nonlinear active noise control and its stability condition," Applied Acoustics, vol. 132, pp. 19-25, 2018.

[33] M. M. Halimeh, C. Huemmer, and W. Kellermann, "A neural network-based nonlinear acoustic echo canceller," Institute of Electrical and Electronics Engineers Signal Processing Letters, vol. 26, no. 12, pp. 1827-1831, 2019.

[34] X. Zhang, X. Ren, J. Na, B. Zhang, and H. Huang, "Adaptive nonlinear neuro-controller with an integrated evaluation algorithm for nonlinear active noise systems," Journal of Sound and Vibration, vol. 329, no. 24, pp. 5005-5016, 2010. 
[35] N. L. Thai, X. Wu, J. Na, Y. Guo, N. T. Trung Tin, and P. X. Le, "Adaptive variable step-size neural controller for nonlinear feedback active noise control systems," Applied Acoustics, vol. 116, pp. 337-347, 2017.

[36] R. L. Welch, S. M. Ruffing, and G. K. Venayagamoorthy, "Comparison of feedforward and feedback neural network architectures for short term wind speed prediction," in Proceedings of the International Joint Conference on Neural Networks, pp. 3335-3340, Budapest, Hungary, July 2009.

[37] M. Salmasi, H. Mahdavi-Nasab, and H. Pourghassem, "Comparison of feed-forward and recurrent neural networks in active cancellation of sound noise," in Proceedings of the International Conference on Multimedia and Signal Processing, vol. 2, pp. 25-29, Shenzhen China, June 2011.

[38] S. Şeker, E. Ayaz, and E. Türkcan, "Elman's recurrent neural network applications to condition monitoring in nuclear power plant and rotating machinery," Engineering Applications of Artificial Intelligence, vol. 16, no. 7-8, pp. 647-656, 2003.

[39] A. Feuer and E. Weinstein, "Convergence analysis of LMS filters with uncorrelated Gaussian data," Institute of Electrical and Electronics Engineers Transactions on Acoustics, Speech, and Signal Processing, vol. 33, no. 1, pp. 222-230, 1985.

[40] L. Tan and J. Jiang, "Adaptive Volterra filters for active control of nonlinear noise processes," Institute of Electrical and Electronics Engineers Transactions on Signal Processing, vol. 49, no. 8, pp. 1667-1676, 2001.

[41] C.-Y. Chang and D.-R. Chen, "Active noise cancellation without secondary path identification by using an adaptive genetic algorithm," Institute of Electrical and Electronics Engineers Transactions on Instrumentation and Measurement, vol. 59, no. 9, pp. 2315-2327, 2010.

[42] F. Russo and G. L. Sicuranza, "Accuracy and performance evaluation in the genetic optimization of nonlinear systems for active noise control," Institute of Electrical and Electronics Engineers Transactions on Instrumentation and Measurement, vol. 56, no. 4, pp. 1443-1450, 2007.

[43] D. C. Le, J. Zhang, D. Li, and S. Zhang, "A generalized exponential functional link artificial neural networks filter with channel-reduced diagonal structure for nonlinear active noise control," Applied Acoustics, vol. 139, pp. 174-181, 2018. 